\title{
MANIFESTAÇÕES CLÍNICAS E LABORATORIAIS DA COVID-19 EM PESSOAS COM DIABETES: REVISÃO INTEGRATIVA
}

\section{COVID-19 CLINICAL AND LABORATORY MANIFESTATIONS IN PEOPLE WITH DIABETES: INTEGRATIVE REVIEW}

\author{
Alex dos Santos Silva ${ }^{1} *$ Caio Bismarck Silva de Oliveira ${ }^{1} *$ Maria Nielly Santos Celestino $^{1}$ \\ Girleide Santos do Nascimento ${ }^{1} *$ Lidiane Lima de Andrade $^{2}$
}

\begin{abstract}
RESUMO
Objetivo: Investigar as evidências científicas sobre as manifestações clínicas e laboratoriais mais frequentes da Covid-19 em pessoas que vivem com diabetes mellitus, quando comparadas às pessoas sem diabetes mellitus. Método: Revisão integrativa da literatura, realizada de 18 de junho a 18 de julho de 2020, baseada na questão norteadora: quais as manifestações clínicas e laboratoriais da Covid-19 em pessoas que vivem com diabetes, quando comparadas às pessoas sem diabetes? Utilizaram-se das bases de dados: Medline, Pubmed, Science Direct e CINAHL, cruzando-se os descritores: "diabetes mellitus" AND "coronavirus infections" e "diabetes mellitus" AND "covid-19". Incluíram-se artigos publicados nos últimos dois anos, que responderam à questão de pesquisa e apresentaram dados passíveis de comparação entre pessoas com e sem diabetes. Excluíram-se artigos de reflexão, relatos de experiência, cartas/comentários ao editor, revisões integrativas de literatura, dissertações, teses e editoriais de periódicos sem caráter científico. Resultados: Na amostra final, obteve-se que os artigos inseridos apresentaram nível de evidência 4. Os dados extraídos dos artigos sugeriram que as manifestações clínicas mais comuns entre os pacientes diabéticos com Covid-19, quando comparados com aqueles que não tinham diabetes, foram tosse, dispneia, fadiga, mialgia e anorexia. Com relação aos dados laboratoriais, notaram-se diferença entre as medianas e aumento nos valores dos neutrófilos, proteína C-reativa, procalcitonina, interleucina 6 e ddímero. Conclusão: A associação do diabetes com a Covid-19 torna os pacientes mais suscetíveis a cursarem a fase grave da infeção, até então, evidenciada pela exacerbação da resposta inflamatória.
\end{abstract}

Palavras-chave: Diabetes Mellitus. Infecções por Coronavirus. Sinais e Sintomas. Biomarcadores.

\begin{abstract}
Objective: To investigate the evidence on the most frequent clinical and laboratory manifestations of covid-19 in people living with diabetes mellitus when compared to people without diabetes mellitus. Methods: Integrative literature review, carried out from June 18 to July 18, 2020, based on the following guiding question: what are the clinical and laboratory manifestations of covid-19 in people living with diabetes when compared to people without diabetes? The following databases were used: Medline, Pubmed, Science Direct and CINAHL, crossing the descriptors: "diabetes mellitus" AND "coronavirus infections" and "diabetes mellitus" AND "covid-19". Articles published in the past two years were included, which answered the research question and presented data that could be compared between people with and without diabetes. Reflection articles, experience reports, letters / comments to the editor, integrative literature reviews, dissertations, theses and editorials of journals without scientific character were excluded. Results: In the final sample, it was found that all articles had evidence level 4. The data extracted from the articles suggested that the most common clinical manifestations among diabetic patients with covid-19 when compared with those who did not have diabetes were cough, dyspnoea, fatigue, myalgia and anorexia. Regarding laboratory data, a difference was noted between the medians and an increase in the values of neutrophils, c-reactive protein, procalcitonin, interleukin 6 and d-dimer. Conclusion: It was found that the association of diabetes and covid-19 made patients more susceptible to undergoing the severe phase of the infection, until then, evidenced by the exacerbation of the inflammatory response.
\end{abstract}

Keywords: Diabetes Mellitus. Coronavirus Infections. Signs and Symptoms. Biomarkers.

\footnotetext{
${ }^{1}$ Discente do curso de Enfermagem da Universidade Federal de Campina Grande, campus Cuité-PB, Brasil.

${ }^{2}$ Enfermeira. Doutora em enfermagem. Docente do curso de Enfermagem da Universidade Federal de Campina Grande, campus Cuité-PB, Brasil. E-mail: lidiane.lima@ufcg.edu.br.
} 


\section{INTRODUÇÃO}

A Organização Mundial de Saúde (OMS) declarou que a coronavirus disease-19 (Covid19), provocada pela Severe Acute Respiratory Syndrome Coronavirus 2 (SARS-CoV-2), constitui emergência de saúde pública de interesse internacional, tendo sido declarada como pandemia, em virtude da gravidade da situação, para que os países passassem a agir efetivamente na detecção de infecções, impedindo a propagação ${ }^{(1)}$.

De acordo com a OMS, em 15 de julho de 2020, o mundo ultrapassava a marca de 13 milhões de casos confirmados e, aproximadamente, 580 mil mortes por Covid$19^{(2)}$. A nível nacional, registraram-se 1,9 milhão de casos da doença, com mais de 74 mil óbitos $^{(3)}$.

O quadro clínico da Covid-19 varia de sintomas brandos, como febre, tosse, cansaço e pneumonia assintomática, a situações mais graves, como síndrome do desconforto respiratório agudo, choque séptico e falência de órgãos, podendo ocasionar a morte ${ }^{(4)}$.

Relatório dos Estados Unidos revelou que $78 \%$ de 457 internações em unidades de terapia intensiva ocorreram em pacientes idosos ou com comorbidades existentes, como doenças pulmonares crônicas, doenças cardiovasculares e Diabetes Mellitus $(\mathrm{DM})^{(5)}$. Frente a isso, estudos vêm tentando evidenciar os perfis clínicos mais suscetíveis a desenvolver o curso grave da Covid-19, apontando o DM como risco potencial.

O DM é uma doença crônica não transmissível, marcada pelo distúrbio do metabolismo da glicose, sendo um dos desafios de saúde mais crescentes. No Brasil, o número de pessoas que vivem com diabetes é elevado. No ano de 2019, a Federação Internacional de Diabetes estimou que 16,8 milhões de pessoas, com idades entre 20 e 79 anos, vivem com a doença, com projeção de aumento de $55 \%$ até $2045^{(6)}$. Deste modo, o maior risco que esse grupo possui para infecções gerais, resultante de múltiplas perturbações da imunidade inata, justifica a necessidade de autoridades de saúde entrarem em alerta.

Os níveis de glicose no sangue são afetados quando os pacientes com diabetes são acometidos por infecções virais em geral, essa flutuação acentuada afeta significativamente o processo de recuperação. Desta forma, com o sistema imunológico desregulado, há maior probabilidade da ocorrência de problemas pulmonares graves, consequente de menor resposta linfocitária e menor número de neutrófilos recrutados para o local da infecção, com a quimiotaxia prejudicada, além de alterações da resposta humoral $^{(7)}$. 
Até o momento, não há evidências que comprovem que os diabéticos sejam mais suscetíveis à infecção pela Covid-19(8). No entanto, nos diabéticos, principalmente aqueles que apresentam alterações glicêmicas, a manifestação inicial da Covid-19 pode ser mais branda, a febre menos comum, e a deterioração pode ocorrer rapidamente em estágios posteriores, como a diminuição na saturação de oxigênio que resulta na hipóxia e, como consequência, na falência de órgãos ${ }^{(9-10)}$.

Todavia, devido à magnitude da doença e à demanda de profissionais na linha de frente, o tempo destinado a pesquisas observacionais se torna reduzido, a maioria das pesquisas disponíveis se limitam a um pequeno número de amostra, com resultados heterogêneos e conflitantes $^{(11)}$. Assim, é imprescindível a identificação dos sinais clínicos e laboratoriais nesse grupo, para se obter o diagnóstico precoce e o monitoramento, que visem controlar a rápida evolução, por meio do tratamento adequado, que minimize os danos físicos e gastos públicos, evitando, também, a superlotação dos serviços de saúde e, por consequência, a mortalidade.

Portanto, para o desenvolvimento deste estudo, delineou-se a questão de pesquisa: quais as manifestações clínicas e laboratoriais da Covid-19 em pessoas que vivem com diabetes, quando comparadas às pessoas sem diabetes?

\section{OBJETIVOS}

Investigar as evidências científicas sobre as manifestações clínicas e laboratoriais mais frequentes da Covid-19 em pessoas que vivem com diabetes mellitus, quando comparadas às pessoas sem diabetes mellitus.

\section{METODOS}

Trata-se de revisão integrativa de literatura, realizada conforme as etapas: 1) elaboração da questão de pesquisa; 2) busca na literatura; 3) avaliação dos dados; 4) análise dos dados - divisão, exposição e comparação; 5) apresentação dos resultados ${ }^{(12)}$.

A busca na literatura ocorreu de 18 de junho a 18 de julho de 2020. A questão de pesquisa foi construída embasada na estratégia PICo (P- população: pessoas com diabetes mellitus; I- Interesse: manifestações clínicas e laboratoriais da Covid-19; Co- Contexto: comparação das pessoas com e sem diabetes), o que resultou na questão norteadora: quais as manifestações clínicas e laboratoriais da Covid19 em pessoas que vivem com diabetes, quando comparadas às pessoas sem diabetes?

As bases de dados utilizadas foram: Medical Literature Analysis and Retrieval System Online (Medline/Pubmed), Pubmed 
Central (PMC), Science Direct e Cumulative Index to Nursing and Allied Health Literature (CINAHL).

Os descritores utilizados foram: diabetes mellitus, coronavirus infections (infecções por coronavírus) e Covid-19, como palavra sinônima, consultados nos Descritores em Ciências da Saúde (DeCS) e no Medical Subject Headings (MeSH). Realizou-se o cruzamento em pares, utilizando-se do operador booleano $A N D$, a saber: "diabetes mellitus" $A N D$ "coronavirus infections" e "diabetes mellitus" AND "covid-19". Para as bases de dados, adotaram-se as seguintes expressões de busca: (diabetes mellitus) AND (coronavirus infections); (diabetes mellitus) AND (covid-19).

Os critérios de inclusão dos estudos foram: responder à questão de pesquisa, publicações dos últimos dois anos (2019-2020) para conhecimento das evidências mais atualizadas sobre a temática em questão e artigos que apresentassem dados passíveis de comparação entre pessoas com e sem diabetes. Excluíram-se artigos de reflexão, relatos de experiência, cartas/comentários ao editor, revisões integrativas de literatura, dissertações, teses e editoriais de periódicos sem caráter científico.

A busca dos artigos foi realizada de forma independente, por dois pesquisadores. Primeiro, procedeu-se à leitura dos títulos e resumos, com a seleção criteriosa dos artigos, conforme os critérios de elegibilidade. Posteriormente, os trabalhos selecionados na etapa anterior foram lidos na íntegra. Por fim, esses estudos foram relidos e analisados, conforme os critérios de elegibilidade, para então, selecionar as publicações que compuseram a amostra final. Nesse processo de amostragem, os resultados dos pesquisadores foram comparados e as diferenças solucionadas por consenso ou com a inclusão de um terceiro revisor, quando necessário, visando favorecer a validação da seleção dos estudos para análise. A busca nas bases de dados resultou em 1.177 publicações, as quais foram selecionadas pelos critérios de elegibilidade, resultando na amostra final de sete artigos (Figura 1). 


\section{PERCURSO DE SELEÇÃO DOS ESTUDOS}

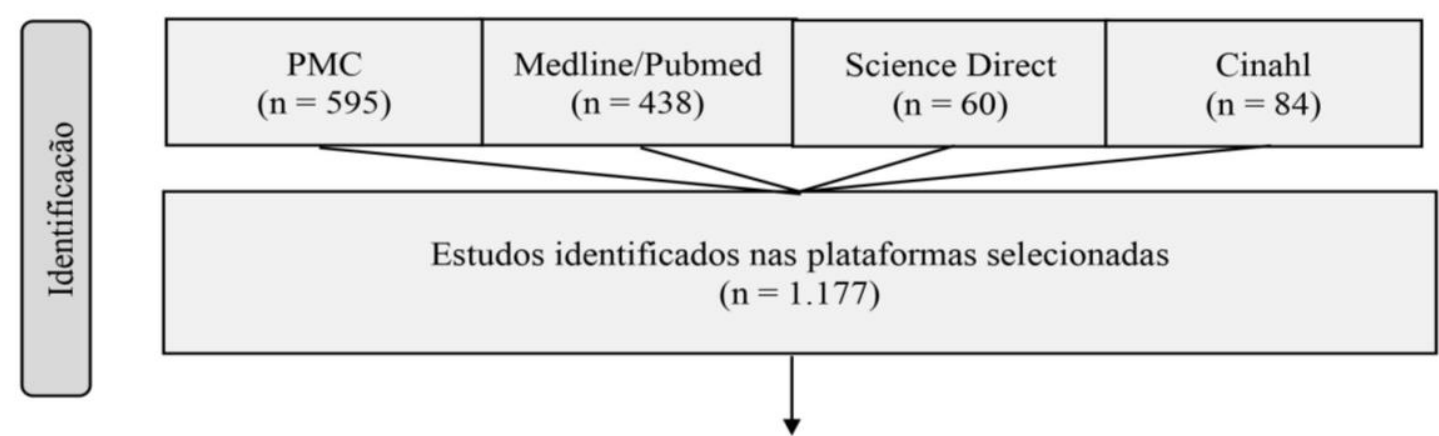

Avaliação dos dados (Artigos selecionados após leitura de títulos e resumos)

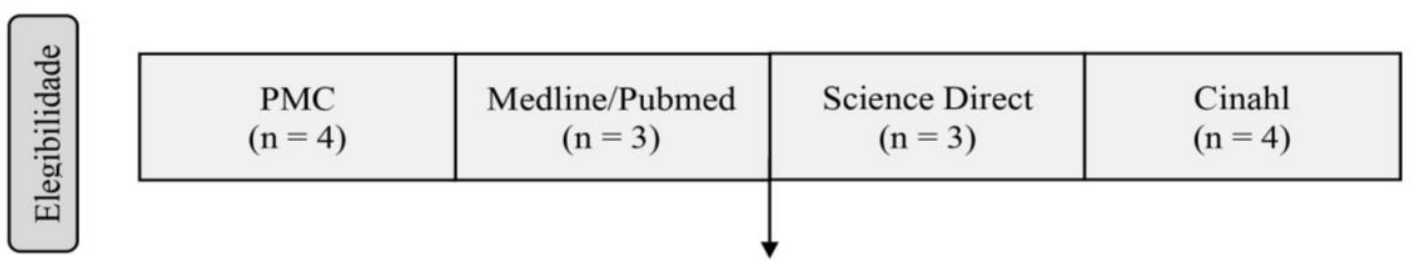

Análise, interpretação e apresentação dos dados (artigos selecionados após leitura na íntegra)

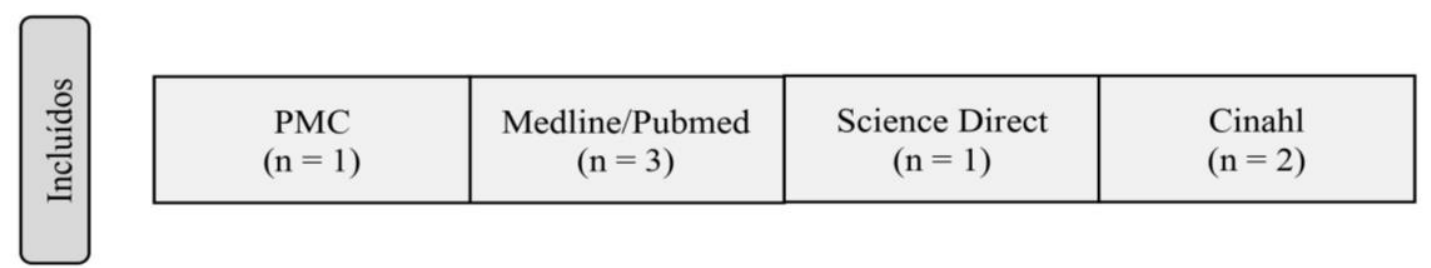

FIGURA 1 - Fluxograma de seleção dos estudos, segundo o Preferred Reporting Items for Systematic Reviews and Meta Analyses (PRISMA). Cuité, Paraíba, Brasil, 2020.

Para coleta de dados dos artigos da amostra final, utilizou-se formulário adaptado a esta pesquisa, contendo dados relacionados aos títulos, autores, anos de publicação, países de desenvolvimento do estudo, às características metodológicas, níveis de evidência, às manifestações clínicas e aos resultados laboratoriais.

O nível de evidência dos estudos foram avaliados pela abordagem metodológica, baseada nas recomendações da Agency for Healthcare Research and Quality (AHRQ) $)^{(13)}$, a qual sugere que a qualidade das evidências científicas estejam categorizadas em: nível 1, metanálise de múltiplos ensaios clínicos controlados e randomizados; nível 2, estudo individual com delineamento experimental; nível 3, estudos 


\section{TIPO DE ARTIGO}

quase experimentais; nível 4, estudos descritivos (não experimentais) ou abordagem qualitativa; nível 5 , relatos de caso ou experiência; nível 6 , opinião de especialistas.

Para análise dos dados, optou-se pela apresentação de forma quantitativa. Assim, na análise bivariada, incialmente, calculouse a Razão de Risco (RR), com os respectivos Intervalos de Confiança (IC) de 95\%, por meio do Teste Qui-quadrado de Pearson. Para associar as manifestações clínicas de pacientes com e sem diabetes, apresentadas nos estudos, valeu-se do programa de domínio público OpenEpi, versão 3.01. Posteriormente, procedeu-se ao levantamento dos resultados laboratoriais mais frequentes de pacientes com e sem diabetes observados nos estudos, sendo apresentados segundo mediana e intervalos interquartílicos. Assim, em detrimento da natureza dos dados, aplicou-se o Teste $\mathrm{U}$ de
Mann-Whitney, mediante software Statistical Package for the Social Sciences, versão 22. Nas análises, o nível de significância adotado foi $<0,05$.

\section{RESULTADOS}

Obteve-se amostra final de sete artigos, com dados de 2.585 pessoas com Covid-19, destas, 582 apresentavam diabetes. Observou-se que os artigos selecionados foram publicados no ano de 2020, sendo três $(42,9 \%)$ destes indexados na base de dados Medline/Pubmed. Ademais, constatou-se que os estudos foram desenvolvidos em Wuhan, na China. Conforme síntese apresentada no Quadro 1, os estudos foram retrospectivos e, por conseguinte, apresentaram nível de evidência igual a 4 .

QUADRO 1 -Síntese dos estudos incluídos nesta revisão integrativa. Cuité, Paraíba, Brasil, 2020.

\begin{tabular}{|c|c|c|}
\hline $\begin{array}{c}\text { Autores, anos, } \\
\text { periódicos, níveis de } \\
\text { evidência }\end{array}$ & $\begin{array}{l}\text { Características metodológicas } \\
\text { (tipo de estudo, local e amostra) }\end{array}$ & Principais resultados \\
\hline $\begin{array}{l}\text { Chen Y et al., } 2020^{(14)} \text {. } \\
\text { Diabetes Care. Nível } \\
\text { de evidência }=4\end{array}$ & $\begin{array}{l}\text { Estudo retrospectivo, realizado no } \\
\text { Hospital Central de Wuhan, China, com } \\
904 \text { pacientes admitidos entre } 15 \text { e } 99 \\
\text { anos com Covid-19, destes, } 136 \text { tinham } \\
\text { diabetes, principalmente tipo } 2 .\end{array}$ & $\begin{array}{l}\text { Os resultados indicaram que } \\
\text { ocorreram mais fatalidades entre } \\
\text { pacientes com diabetes e Covid-19 } \\
\text { do que naqueles com Covid-19 } \\
\text { sem diabetes. }\end{array}$ \\
\hline $\begin{array}{l}\text { Shi Q et al., } 2020^{(15)} \text {. } \\
\text { Diabetes } \text { Care. Nível } \\
\text { de evidência }=4\end{array}$ & $\begin{array}{l}\text { Estudo retrospectivo, realizado em dois } \\
\text { hospitais terciários em Wuhan, China, } \\
\text { sendo coletados dados de } 306 \text { pacientes }\end{array}$ & $\begin{array}{l}\text { Os resultados apontaram que } \\
\text { pacientes com diabetes tinham } \\
\text { mais comorbidades subjacentes, }\end{array}$ \\
\hline
\end{tabular}




\begin{tabular}{|c|c|c|}
\hline & $\begin{array}{l}\text { com Covid-19, destes, } 153 \text { pacientes } \\
\text { viviam com diabetes. }\end{array}$ & $\begin{array}{l}\text { eram mais propensos a sofrer } \\
\text { complicações, tiveram proporção } \\
\text { maior de admissões na Unidade de } \\
\text { Terapia Intensiva, e mais mortes } \\
\text { em comparação com pacientes da } \\
\text { mesma idade e sexo sem diabetes. }\end{array}$ \\
\hline $\begin{array}{l}\text { Yan Y et al., } 2020^{(16)} \text {. } \\
\text { BMJ Open Diabetes } \\
\text { Research \& Care. } \\
\text { Nível de evidência=4 }\end{array}$ & $\begin{array}{l}\text { Estudo retrospectivo, desenvolvido no } \\
\text { Hospital Tongji de Wuhan, China, com } \\
193 \text { pacientes que tinham Covid-19, } \\
\text { destes, } 48 \text { tinham diabetes. }\end{array}$ & $\begin{array}{l}\text { Os resultados sugeriram que } \\
\text { pacientes com Covid-19 e diabetes } \\
\text { eram mais propensos a receber } \\
\text { ventilação mecânica e admissão na } \\
\text { Unidade de Terapia Intensiva, } \\
\text { também apresentavam mortalidade } \\
\text { mais elevada e exibiram resposta } \\
\text { inflamatória grave. }\end{array}$ \\
\hline $\begin{array}{l}\text { Zhang Y et al., } 2020^{(17)} \text {. } \\
\text { Diabetes Research and } \\
\text { Clinical Practice. Nível } \\
\text { de evidência }=4\end{array}$ & $\begin{array}{l}\text { Estudo retrospectivo produzido no West } \\
\text { Court of Union Hospital, em Wuhan, } \\
\text { China, com } 258 \text { pacientes hospitalizados } \\
\text { com Covid-19, destes, } 63 \text { tinham diabetes. }\end{array}$ & $\begin{array}{l}\text { Os resultados estimaram que o } \\
\text { diabetes mellitus esteve associado } \\
\text { a uma maior gravidade da Covid- } \\
19 \text { e a piores desfechos em curto } \\
\text { prazo, incluindo morte. }\end{array}$ \\
\hline $\begin{array}{l}\text { Shang J et al., } 2020^{(18)} \text {. } \\
\text { The American journal } \\
\text { of medicine. Nível de } \\
\text { evidência }=4\end{array}$ & $\begin{array}{l}\text { Estudo retrospectivo, desenvolvido no } \\
\text { Hospital Zhongnan de Wuhan University, } \\
\text { em Wuhan, China, com } 584 \text { pacientes } \\
\text { com Covid-19, destes, } 84 \text { tinham diabetes. }\end{array}$ & $\begin{array}{l}\text { Os resultados concluíram que } \\
\text { apesar de receber tratamento } \\
\text { avançado, a mortalidade dos } \\
\text { pacientes no grupo com diabetes e } \\
\text { Covid-19, ainda, era } \\
\text { significativamente maior do que } \\
\text { no grupo sem diabetes. }\end{array}$ \\
\hline $\begin{array}{l}\text { Guo W et al., } 2020^{(10)} \\
\text { Diabetes/Metabolism } \\
\text { Research and Reviews. } \\
\text { Nível de evidência=4 }\end{array}$ & $\begin{array}{l}\text { Estudo retrospectivo, desenvolvido no } \\
\text { Wuhan Union Hospital, em Wuhan, } \\
\text { China, com } 174 \text { pacientes com Covid-19, } \\
\text { destes, } 37 \text { tinham diabetes. }\end{array}$ & $\begin{array}{l}\text { Os resultados demonstraram que } \\
\text { independentemente de haver ou } \\
\text { não interferência de outras } \\
\text { comorbidades, pacientes com } \\
\text { pneumonia por SARS-CoV-2 com } \\
\text { diabetes são mais graves do que } \\
\text { aqueles sem diabetes, avaliados } \\
\text { por danos a órgãos, fatores } \\
\text { inflamatórios ou } \\
\text { hipercoagulabilidade, e com maior } \\
\text { probabilidade de progredir para } \\
\text { um pior prognóstico. }\end{array}$ \\
\hline $\begin{array}{l}\text { Zhang Y et al., } 2020^{(19)} \text {. } \\
\text { Diabetes, Obesity and } \\
\text { Metabolism. Nível de } \\
\text { evidência }=4\end{array}$ & $\begin{array}{l}\text { Estudo retrospectivo elaborado no Tongji } \\
\text { Hospital in the Zhongfa, em Wuhan, } \\
\text { China, com } 166 \text { pacientes com Covid-19, } \\
\text { destes, } 61 \text { tinham diabetes. }\end{array}$ & $\begin{array}{l}\text { Os resultados inferiram que a } \\
\text { maior gravidade da doença e taxa } \\
\text { de mortalidade em pacientes com } \\
\text { Covid-19 e diabetes, pode ser } \\
\text { resultado de comorbidades } \\
\text { crônicas, resposta imunológica } \\
\text { fraca e maior risco de infecções } \\
\text { bacterianas secundárias. }\end{array}$ \\
\hline
\end{tabular}

$\mathrm{Na}$ Tabela 1, observa-se que as manifestações clínicas contidas nos estudos: 


\section{TIPO DE ARTIGO}

tosse, dispneia, fadiga, mialgia e anorexia, apresentaram diferença estatisticamente

significante entre pessoas com e sem diabetes, com o diagnóstico de Covid-19.

TABELA 1- Associação das manifestações clínicas da Covid-19 em pacientes com e sem diabetes, de acordo com a literatura. Cuité, Paraíba, Brasil, 2020.

\begin{tabular}{|c|c|c|c|c|c|c|}
\hline \multicolumn{2}{|c|}{ Manifestações clínicas } & \multirow{2}{*}{$\begin{array}{c}\text { Com } \\
\text { Diabetes } \\
\text { n (\%) } \\
427(22,8)\end{array}$} & \multirow{2}{*}{$\begin{array}{c}\begin{array}{c}\text { Sem } \\
\text { Diabetes } \\
\text { n }(\%)\end{array} \\
1441(77,2)\end{array}$} & \multirow{2}{*}{$\begin{array}{c}\text { p-valor } \\
0,292\end{array}$} & \multirow{2}{*}{$\begin{array}{c}\mathbf{R R} \\
0,95\end{array}$} & \multirow{2}{*}{$\begin{array}{c}\text { IC a } \mathbf{9 5 \%} \\
0,80-1,12\end{array}$} \\
\hline Febre & Sim & & & & & \\
\hline & Não & $145(24,0)$ & $458(76,0)$ & & 1 & \\
\hline \multirow{2}{*}{ Tosse } & Sim & $364(25,0)$ & $1091(75,0)$ & $<0,001$ & 1,26 & $1,08-1,46$ \\
\hline & Não & $220(19,8)$ & $889(80,2)$ & & 1 & \\
\hline \multirow[t]{2}{*}{ Dispneia } & Sim & $201(29,5)$ & $480(70,5)$ & 0.024 & 1.17 & $1,00-1,38$ \\
\hline & Não & $245(25,0)$ & $734(75,0)$ & & 1 & \\
\hline \multirow[t]{2}{*}{ Fadiga } & Sim & $272(29,5)$ & $649(97,5)$ & $<0,001$ & 1,56 & $1,35-1,80$ \\
\hline & Não & $310(18,9)$ & $1333(81,1)$ & & 1 & \\
\hline \multirow[t]{2}{*}{ Mialgia } & Sim & $73(32,7)$ & $150(67,3)$ & 0.004 & 1.35 & $1,09-1,67$ \\
\hline & Não & $325(24,2)$ & $1019(75,8)$ & & 1 & \\
\hline \multirow[t]{2}{*}{ Cefaleia } & Sim & $30(26,3)$ & $84(73,7)$ & 0.056 & 0.76 & $0,55-1,05$ \\
\hline & Não & $329(34,2)$ & $633(65,8)$ & & 1 & \\
\hline \multirow[t]{2}{*}{ Diarreia } & Sim & $84(27,3)$ & $223(72,7)$ & 0.403 & 0,96 & $0,79-1,18$ \\
\hline & Não & $362(28,3)$ & $919(71,7)$ & & 1 & \\
\hline \multirow{2}{*}{ Náuseas/Vômito } & Sim & $65(29,1)$ & $158(70,9)$ & 0.479 & 1,00 & $0,80-1,24$ \\
\hline & Não & $553(29,1)$ & $1350(70,9)$ & & 1 & \\
\hline \multirow[t]{2}{*}{ Anorexia } & Sim & $164(32,0)$ & $349(68,0)$ & 0.007 & 1,25 & $1,05-1,50$ \\
\hline & Não & $182(25,5)$ & $533(74,5)$ & & 1 & \\
\hline
\end{tabular}

$\mathrm{Na}$ Tabela 2, os dados apontam diferenças entre as medianas dos resultados laboratoriais: neutrófilos, proteína C-reativa, procalcitonina, interleucina 6 e ferritina diferença estatisticamente significante entre pessoas com e sem diabetes, com o diagnóstico de Covid-19.

TABELA 2- Comparação dos resultados laboratoriais mais frequentes em pacientes com Covid-19, com e sem diabetes. Cuité, Paraíba, Brasil, 2020.

\begin{tabular}{lccc}
\hline \multicolumn{1}{c}{$\begin{array}{c}\text { Exames } \\
\text { Laboratoriais }\end{array}$} & $\begin{array}{c}\text { Com diabetes } \\
\text { Mediana (Q25-75) }\end{array}$ & $\begin{array}{c}\text { Sem diabetes } \\
\text { Mediana (Q25-75) }\end{array}$ & p-valor \\
\hline Neutrófilos & $4,40(3,86-4,60)$ & $3,30(3,23-3,82)$ & 0,004 \\
Linfócitos & $0,90(0,84-1,08)$ & $1,04(0,90-1,10)$ & 0,259 \\
Proté́na C-reativa & $32,80(25,10-36,10)$ & $16,80(15,50-30,60)$ & 0,038 \\
Procalcitonina & $0,09(0,07-0,09)$ & $0,06(0,05-0,07)$ & 0,026 \\
Interleucina-6 & $23,00(15,90-23,00)$ & $12,55(11,10-12,90)$ & 0,038 \\
\hline
\end{tabular}




\begin{tabular}{lccc}
\hline Ferritina sérica & $904,13(739,60-904,13)$ & $504,30(504,30-509,80)$ & 0,001 \\
D-dímero & $0,80(0,60-1,80)$ & $0,50(0,50-0,80)$ & 0,128 \\
\hline
\end{tabular}

\section{DISCUSSÃO}

Os achados desta pesquisa apontaram a concentração de estudos retrospectivos em Wuhan, na China. Este dado se deve ao fato de que a doença foi identificada pela primeira vez nesta cidade. Acrescenta-se a isso o número de estudos observacionais ainda reduzidos, assim, aponta-se a necessidade de mais estudos sobre esta temática.

No que diz respeito às manifestações clínicas, de acordo com a sumarização dos dados, infere-se que as pessoas com diabetes tinham 1,2 vezes mais risco de terem tosse, quando comparadas às pessoas sem diabetes. As pessoas com diabetes tinham o 1,1 vezes mais risco de terem dispneia, quando comparadas às pessoas sem diabetes. As pessoas com diabetes tinham 1,5 vezes mais risco de apresentarem fadiga, quando confrontadas com as pessoas sem diabetes. As pessoas com diabetes tinham 1,3 vezes mais risco de terem mialgia, quando equiparadas às pessoas sem diabetes. $\mathrm{E}$ as pessoas com diabetes tinham 1,2 vezes mais risco de terem anorexia, em relação às pessoas sem diabetes.

Em coorte retrospectiva, realizada na
China, com objetivo de investigar o controle da glicose no sangue, dividindo os diabéticos entre bem controlados e mal controlados, estimou-se que as manifestações clínicas mais frequentes foram fadiga e dispneia entre os usuários que tinham Covid-19 e diabetes $^{(20)}$, corroborando os dados deste estudo.

Outros estudos, desenvolvidos com pacientes infectados pelo SARS- CoV-2, apontam como manifestação clínica mais frequente a febre, fadiga e tosse ${ }^{(4,21)}$.

No tocante à febre, estudo retrospectivo sugeriu que a presença de DM, temperatura corporal $\geq 37,8^{\circ} \mathrm{C}$ e saturação periférica de oxigênio < $92 \%$ são fatores preditores da forma grave da doença em pacientes hospitalizados com Covid-19, sendo o monitoramento importante para fornecer intervenções necessárias a pacientes pertencentes ao grupo de risco ${ }^{(9)}$.

A mialgia também foi um sinal clínico observado entre os diabéticos. A dor muscular surge em virtude do aumento dos níveis de lactato desidrogenase, diminuição do pH e oxigenação, estes, por sua vez, são alterados devido ao dano causado pelo vírus no músculo e em outros tecidos. Quando a 
carga do vírus diminui, a oxigenação dos eritrócitos aumenta e o nível de lactato muscular diminui, amenizando, assim, a mialgia $^{(22)}$.

Outro sinal clínico observado em pacientes diabéticos foi a anorexia. Verificou-se que pacientes acometidos por Covid-19 e que vivem com diabetes, que alterações nos hábitos alimentares passaram a apresentar variações nos níveis de glicose no sangue ${ }^{(23)}$. Estudos realizados no Hospital de Tongji, em Wuhan, sugerem que a ingesta de alimentos se torna prejudicada em casos mais graves de Covid-19, visto que os pacientes passaram a contar com o auxílio de um suporte nutricional, por infusão intravenosa de líquidos. Além disso, a infecção por coronavírus pode causar disfunção da absorção gastrointestinal ${ }^{(16)}$.

Com relação aos exames laboratoriais, destaca-se aumento na mediana dos valores de todos resultados, com exceção dos linfócitos, visto que há linfopenia mais acentuada em pessoas com diabetes, quando comparadas às pessoas sem diabetes.

O SARS-CoV-2 se espalha pela mucosa e infecta outras células, induzindo a liberação de tempestade de citocinas no corpo, gerando resposta imune e causando alteração de leucócitos periféricos e nas células imunes, como os linfócitos e neutrófilos. Embora a hiperglicemia induza à diminuição da quimiotaxia dos neutrófilos, em pacientes diabéticos foi observado aumento do número de neutrófilos, devido à tempestade de citocinas causada pelo vírus $^{(24-25)}$.

Estudos observaram que essa tempestade gera um estado hiper inflamatório e ocasiona maior destruição tecidual, ocasionada pela liberação de enzimas, comprometendo órgãos como pulmão, coração e rins ${ }^{(10,26)}$. Deste modo, sugere-se que o aumento da gravidade da doença nos pacientes diabéticos ocorre devido à alteração da resposta imune que gera resposta inflamatória exacerbada, acarretando patologia pulmonar mais grave e prolongada ${ }^{(27)}$.

Ao analisar os achados laboratoriais disponíveis na literatura, verificou-se que os pacientes com DM possuem aumento da Proteína C-reativa, em virtude do quadro inflamatório exacerbado, além disso, há também o aumento da Interleucina-6, que equiparada às demais citocinas, apresenta tempo de expressão significativamente maior, gerando estado de inflamação prolongada $^{(10)}$. Também se obteve aumento 
da Procalcitonina, biomarcador importante para identificar o risco de infecção generalizada $^{(28)}$.

Ademais, observou-se que entre os pacientes com DM existe o aumento da Ferritina sérica que, por sua vez, consiste em proteína de armazenamento de ferro nos tecidos. Os níveis elevados são característicos de inflamações e estimulação imunológica, uma vez que que esta é importante biomarcador do processo inflamatório, ativando o sistema monócitomacrófago na tempestade inflamatória ${ }^{(10,29)}$.

Embora sem diferença estatística entre os grupos, os achados apontaram que os diabéticos possuíam nível maior do Ddímero nos resultados laboratoriais, sugerindo, assim, que essa população está mais susceptível a desenvolver um estado hipercoagulável do que os pacientes não diabéticos, essa ativação de coagulação pode estar relacionada à resposta inflamatória sustentada $^{(20,24)}$.

O D-dímero é um dos principais marcadores da atividade de coagulação, sendo originado a partir da fibrina ${ }^{(30)}$. No estágio inicial, o dímero é o resultado da inflamação que ativa a plasmina. À medida que a inflamação progride, as moléculas induzidas por hipóxia podem ativar a trombina diretamente, ocorrendo a ativação de macrófagos e monócitos, ocasionando a secreção de uma massa de fatores teciduais e ativaria a via de coagulação exógena, que resultaria em estado de hipercoagulável geral ou coagulação intravascular disseminada ${ }^{(10)}$.

Esses achados sugerem que o DM, associado à infecção viral pelo SARS-CoV-2, torna o quadro clínico e laboratorial mais acentuado, com mal prognóstico. É importante identificar tais aspectos preditores, para que, com base no conhecimento teórico, a prática profissional possa ser direcionada corretamente, estando atento aos sinais e sintomas, a fim de entender a gravidade do quadro inflamatório e buscar minimizar os danos ocasionados ${ }^{(31)}$. Enfatiza-se, também, a relevância de monitorar os níveis glicêmicos, visto que esses pacientes possuem resposta imunológica alterada, sendo propensos a desenvolver quadro inflamatório exacerbado. Como também, avaliar a saturação de oxigênio, visando evitar deterioração de órgãos causados pela hipóxia prolongada ${ }^{(32)}$.

Com base no exposto, evidencia-se que a assistência integral, prestada de forma eficiente e com base no respaldo científico, permite identificar detalhes importantes para intervenção necessária, evitando evoluções 


\section{TIPO DE ARTIGO}

inesperadas que acarretem o desenvolvimento de quadros graves, até mesmo o óbito.

Quanto às limitações desta pesquisa, destaca-se o pequeno quantitativo amostral de estudos analisados, o que desperta a necessidade de mais estudos, principalmente de caráter observacional, para evidência de dados clínicos e laboratoriais mais precisos e representativos.

\section{CONCLUSÃO}

Os dados extraídos dos artigos sugeriram que as manifestações clínicas mais comuns entre os pacientes diabéticos com Covid-19, quando comparados com aqueles que não tinham diabetes, foram tosse, dispneia, fadiga, mialgia e anorexia.

Com relação aos dados laboratoriais entre os pacientes diabéticos com Covid-19, comparados com aqueles que não tinham diabetes, constataram-se diferença entre as medianas e aumento nos valores dos neutrófilos, proteína C-reativa, procalcitonina, interleucina 6 e d-dímero. Portanto, verificou-se que a associação de diabetes e Covid-19 torna os pacientes mais suscetíveis a cursarem a fase grave da infeção, até então, evidenciada pela exacerbação da resposta inflamatória.

\section{REFERÊNCIAS}

1. Güner R, Hasanoğlu I, Aktas F. COVID19: Prevention and control measures in community. Turkish Journal of Medical Sciences [Internet]. 2020 [acesso em 17 jun 2020]; 50(3): 571-577. Disponível em: https://www.ncbi.nlm.nih.gov/pmc/articles/P MC7195988/.

2. World Health Organization. WHO Coronavirus Disease (COVID-19) Dashboard [Internet]. Switzerland; 2020 [acesso em 15 jul 2020]. Disponível em: https://covid19.who.int/.

3. Brasil. Ministério da Saúde. Painel Coronavírus [Internet]. Brasília; 2020 [acesso em 15 jul 2020]. Disponível em: https://covid.saude.gov.br/.

4. Guan W, Ni Z, Hu Y, Liang W, Ou C, He $\mathrm{J}$, et al. Clinical Characteristics of Coronavirus Disease 2019 in China. New England Journal of Medicine [Internet]. 2020 [acesso em 17 jun 2020]; 382: 17081720. Disponível em: https://www.nejm.org/doi/full/10.1056/NEJ Moa2002032.

5. Chow N, Fleming-Dutra K, Gierke R, Hall A, Hughes M, Pilishvili $T$, et al. Preliminary Estimates of the Prevalence of Selected Underlying Health Conditions Among Patients with Coronavirus Disease 2019. MMWR Morb Mortal Wkly Rep [Internet]. 2020 [acesso em 18 jun 2020]; 69(13): 382-386. Disponível em: https://www.cdc.gov/mmwr/volumes/69/wr/ mm6913e2.htm\#suggestedcitation.

6. Federação Internacional de Diabetes. Atlas de Diabete da IDF, $9^{a}$ edn. [Internet]. Bruxelas, Bélgica, 2019 [acesso em 18 jun 
2020]. Disponível em: https://diabetesatlas.org/

12. Whittemore R, Knafl K. The integrative review: update methodology. J Adv Nurs.

7. Ma RCW, Holt RIG. COVID-19 e diabetes. Diabet Med [Internet]. 2020 [acesso em 18 jun 2020]; 37 (5): 723725. Disponível em: https://www.ncbi.nlm.nih.gov/pmc/articles/P MC7228343/.

8. Fadini GP, Morieri ML, Longato E, Avogaro A. Prevalence and impact of diabetes among people infected with SARSCoV-2. J Endocrinol Invest [Internet]. 2020 [acesso em 18 jun 2020]; 43(6):867-869. Disponível em: https://www.ncbi.nlm.nih.gov/pmc/articles/P MC7103097/.

9. Jang JG, Hur J, Choi EY, Hong KS, Lee W, Ahn JH. Prognostic Factors for Severe Coronavirus Disease 2019 in Daegu, Korea. J Korean Med Sci [Internet]. 2020 [acesso em 18 jun 2020]; 35(23):e209. Disponível em: https://www.ncbi.nlm.nih.gov/pmc/articles/P $\underline{\text { MC7295599/ }}$

10. Guo W, Li M, Dong Y, Zhou H, Zhang $Z$, Tian C, et al. Diabetes is a risk factor for the progression and prognosis of COVID-19. Diabetes Metab Res Ver [Internet]. 2020 [acesso em 05 jul 2020];1-9. Disponível em: https://onlinelibrary.wiley.com/doi/full/10.1 $\underline{002 / \mathrm{dmrr} .3319}$

11. Kumar A, Arora A, Sharma P, Anikhindi AS, Bansal N, Singla V, et al. Is diabetes mellitus associated with mortality and severity of COVID-19? A meta-analysis. Diabetes \& metabolic syndrome [Internet]. 2020 [acesso em 19 jun 2020];14(4):535-545. Disponível: https://www.ncbi.nlm.nih.gov/pmc/articles/P MC7200339/ [Internet]. 2005 [acesso em 14 jul 2020];52(5):546-53. Disponível em: https://onlinelibrary.wiley.com/doi/epdf/10.1 $\underline{111 / \mathrm{j} .1365-2648.2005 .03621 . \mathrm{x}}$

13. Stetler CB, Morsi D, Rucki S, Broughton $\mathrm{S}$, Corrigan B, Fitsgerald J, Giuliano K, Havener K, Sheridan EA. Utilizationfocused integrative reviews in a nursing service. Appl Nurs Res. [Internet]. 1998 [acesso em 14 jul 2020];11(4):195-206. Disponível em: https://www.sciencedirect.com/science/articl e/pii/S0897189798803297

14. Chen Y, Yang D, Cheng B, Chen J, Peng A, Yang C, et al. Clinical Characteristics and Outcomes of Patients with Diabetes and COVID-19 in Association with GlucoseLowering Medication. Diabetes Care [Internet]. 2020 [acesso em 25 jun 2020];43(7):1399-407. Disponível em: https://care.diabetesjournals.org/content/43/7 $\underline{1399}$

15. Shi Q, Zhang X, Jiang F, Zhang X, Hu N, Bimu C, et al. Clinical Characteristics and Risk Factors for Mortality of COVID-19 Patients With Diabetes in Wuhan, China: A Two-Center, Retrospective Study. Diabetes Care [Internet]. 2020 [acesso em 25 jun 2020]. Disponível em: https://care.diabetesjournals.org/content/43/7 $\underline{1382}$

16. Yan Y, Yang Y, Wang F, Ren H, Zhang $S$, Shi $X$, et al. Clinical characteristics and outcomes of patients with severe covid-19 with diabetes. BMJ Open Diabetes Res Care [Internet]. 2020 [acesso em 26 jun 2020];8(1):1-9. Disponível em: https://drc.bmj.com/content/8/1/e001343 
17. Zhang Y, Cui Y, Shen M, Zhang J, Liu B, Dai M, et al. Association of diabetes mellitus with disease severity and prognosis in COVID-19: A retrospective cohort study. Diabetes Res Clin Pract [Internet]. 2020 [acesso em 27 jun 2020];165:108227. Disponível em: https://www.diabetesresearchclinicalpractice .com/article/S0168-8227(20)30477-0/pdf

18. Shang J, Wang Q, Zhang H, Wang X, Wan J, Yan Y, et al. The Relationship between Diabetes Mellitus and COVID-19 Prognosis: A Retrospective Cohort Study in Wuhan, China. Am J Med [Internet]. 2020 [acesso em 30 jun 2020]; S00029343(20)30532-5. Disponível em: https://www.ncbi.nlm.nih.gov/pmc/articles/P $\underline{\mathrm{MC} 7350644 /}$

19. Zhang Y, Li H, Zhang J. The clinical characteristics and outcomes of patients with diabetes and secondary hyperglycaemia with coronavírus disease 2019: A single-centre, retrospective, observational study in Wuhan. Diabetes Obes Metab [Internet] 2020 [acesso em 07 jul 2020];22:1443-1454. Disponível em: $\quad$ https://dompubs.onlinelibrary.wiley.com/doi/full/10.111 $\underline{1 / \text { dom. } 14086}$

20. Zhu L, She ZG, Cheng X, Qin JJ, Zhang $\mathrm{XJ}$, Cai J, et al. Association of Blood Glucose Control and Outcomes in Patients with COVID-19 and Pre-existing Type 2 Diabetes. Cell Metab [Internet] 2020 [acesso em 29 jun 2020]; 31(6):1068-1077.e3. Disponível em: https://www.ncbi.nlm.nih.gov/pmc/articles/P $\underline{\mathrm{MC} 7252168 /}$

21. Wang D, Hu B, Hu C, Zhu F, Liu X, Zhang J, et al. Clinical Characteristics of 138 Hospitalized Patients With 2019 Novel
Coronavirus-Infected Pneumonia in Wuhan, China. JAMA [Internet] 2020 [acesso em 29 jun 2020]; 323(11):1061-1069. Disponível em:

https://www.ncbi.nlm.nih.gov/pmc/articles/P $\underline{\mathrm{MC} 7042881 /}$

22. Kucuk A, Cumhur Cure M, Cure E. Can COVID-19 cause myalgia with a completely different mechanism? A hypothesis. Clin Rheumatol [Internet]. 2020 [acesso em 18 jul 2020];39(7):2103-4. Disponível em: https://link.springer.com/article/10.1007/s10 067-020-05178-1

23. Barone MTU, Harnik SB, de Luca PV, Lima BL de S, Wieselberg RJP, Ngongo B, et al. The impact of COVID-19 on people with diabetes in Brazil. Diabetes Res Clin Pract [Internet]. 2020 [acesso em 18 jul 2020];166:108304. Disponível em: https://www.diabetesresearchclinicalpractice .com/article/S0168-8227(20)30556-8/pdf

24. Papadokostaki E, Tentolouris N, Liberopoulos E. COVID-19 and diabetes: What does the clinician need to know? Prim Care Diabetes [Internet]. 2020 [acesso em 18 jul 2020]. Disponível em: https://www.primary-carediabetes.com/article/S1751-9918(20)30222$\underline{9 / \mathrm{pdf}}$

25. Gao Y, Li T, Han M, Li X, Wu D, Xu Y, et al. Diagnostic utility of clinical laboratory data determinations for patients with the severe COVID-19. Journal of medical virology.[Internet]. 2020 [acesso em 1 jul 2020]; 92(7): 791-796. Disponível em: https://onlinelibrary.wiley.com/doi/full/10.1 $\underline{002 / \mathrm{jmv} .25770}$

26. Wong CK, Lam CWK, Wu AKL, Lee NLS, Chan IHS, Lit LCW, et al. Plasma inflammatory cytokines and chemokines in 
severe acute respiratory syndrome. Clinical \& Experimental Immunology [Internet]. 2004 [acesso em 1 jul 2020]; 136(1), 95-103. Disponível em: https://onlinelibrary.wiley.com/doi/full/10.1 $\underline{111 / j .1365-2249.2004 .02415 . x}$

27. Kulcsar KA, Coleman CM, Beck SE, Frieman MB. Comorbid diabetes results in imune dysregulation and enhanced disease severity following MERS-CoV infection. JCI Insight [Internet]. 2019 [acesso em 02 jul 2020]; 4(20):e131774. Disponível em: https://www.ncbi.nlm.nih.gov/pmc/articles/P MC6824443/

28. Guven H, Altintop L, Baydin A, Hokelek M, Doganay Z, Bek Y, et al. Diagnostic value of procalcitonin levels as an early indicator of sepsis. The American journal of emergency medicine [Internet]. 2002 [acesso em 03 jul 2020]; 20(3), 202-206. Disponível em:

https://www.ajemjournal.com/article/S0735$\underline{6757(02) 32324-6 / \text { fulltext }}$

29. Wang W, Ann M, Coffman LG, Torti FM, Torti S V. Biochimica et Biophysica Acta Serum ferritin: Past, present and future. BBA - Gen Subj [Internet]. 2010 [acesso em 18 jul 2020];1800(8):760-9. Disponível em: https://www.sciencedirect.com/science/articl e/abs/pii/S0304416510000851?via\%3Dihub

30. Le Gal G, Righini M, Wells PS. D-dimer for pulmonary embolism. JAMA [Internet]. 2015 [acesso em 3 jul 2020]; 313(16):16681669. Disponível em: https://pubmed.ncbi.nlm.nih.gov/25919531/

31. Chen N, Zhou M, Dong X, Qu J, Gong F, Han Y, et al. Epidemiological and clinical characteristics of 99 cases of 2019 novel coronavirus pneumonia in Wuhan, China: a descriptive study. The Lancet [Internet]. 2020 [acesso em 5 jul 2020]; 395(10223), 507-513. Disponível em: https://www.thelancet.com/journals/lancet/ar ticle/PIIS0140-6736(20)30211-7/fulltext

32. Yang JK, Feng Y, Yuan MY, Yuan SY, Fu HJ, Wu BY, et al. Plasma glucose levels and diabetes are independent predictors for mortality and morbidity in patients with SARS. Diabet Med [Internet]. 2006 [acesso em 19 jul 2020];23(6):623-8. Disponível em: https://onlinelibrary.wiley.com/doi/abs/10.11 $\underline{11 / \mathrm{j} .1464-5491.2006 .01861 . \mathrm{x}}$

Recebido: $2020-08-18$

Aceito: 2020-07-24 